\title{
COMPARAÇÃO DOS NÍVEIS DE APTIDÃO FÍSICA ENTRE HIPERTENSOS E NORMOTENSOS
}

\author{
COMPARISON OF LEVELS OF PHYSICAL FITNESS BETWEEN HYPERTENSIVE AND NORMOTENSIVE \\ INDIVIDUALS
}

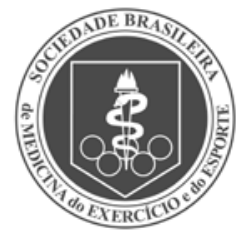

Artigo Original

Original Article

Artículo Original

\section{COMPARACIÓN DELOS NIVELES DEAPTITUD FÍSICA ENTRE HIPERTENSOS Y NORMOTENSOS}

\author{
Lara Belmudes Bottcher ${ }^{1}$ \\ (Profissional de Educão Física) \\ Eduardo Kokubun² \\ (Profissional de Educão Física)
}

1. Centro Universitário Doutor Leão Sampaio; Juazeiro do Norte, CE, Brasil.

2. Universidade Estadual Paulista "Júlio de Mesquita Filho", Institudo de Biociências, Núcleo de Atividade Física, Esporte e Saúde, Departamento de Educação Física, Rio Claro, São Paulo, Brasil.

\section{Correspondência:}

Rua Vicente Ferreira Duarte, 62, Cidade Universitária, Juazeiro do Norte, CE, Brasil. 63048-200. larabottcher@hotmail.com

\section{RESUMO}

Introdução: A hipertensão arterial é um fator de risco de maior importância para morbidade e mortalidade causadas por doenças cardiovasculares. Estudos apontam relação inversa entre taxa de mortalidade e nível de aptidão física. Objetivo: Verificar se existe diferença na aptidão física entre hipertensos e normotensos e se existe efeito da prática regular de atividade física sobre a aptidão física em hipertensos similar às encontradas em normotensos. Métodos: Participaram do estudo 214 mulheres ( $\geq 40$ anos), que fizeram parte, por seis meses, de um programa de atividade física. Todas foram submetidas a uma avaliação antropométrica e avaliação da aptidão física (AAHPERD). Resultados: Hipertensos têm condição inicial pior com relação a agilidade ( $G H$ média inicial $=24,2 \pm 0,4$ seg. $e$ $\mathrm{GN}=20,9 \pm 0,8$ seg., $\mathrm{p}<0,01$ ), coordenação (GH média inicial $=17,5 \pm 0,7$ seg. e $\mathrm{GN}$ média inicial $=11,5 \pm 1,2$ seg., $p<0,01$ ) e resistência aeróbica (GH média inicial $=568,5 \pm 12,2$ seg. e GN média inicial $=506,8 \pm 21,7$ seg. $\mathrm{p}<0,02)$. Após seis meses de prática de atividade física regular, os indivíduos hipertensos continuam apresentando resultados piores quanto a agilidade, coordenação e resistência aeróbica ( $G H$ média 6 meses $=22,9 \pm 0,4$ seg. e GN média 6 meses = 19,97 $\pm 0,7$ seg., p <0,01; GH 6 meses $=16,1 \pm 0,7$ seg. e GN 6 meses = 10,6 $\pm 1,3$ seg., $p<0,01$; $\mathrm{GH} 6$ meses $=498,9 \pm 20,2$ seg. e GN 6 meses $=555,7 \pm 11,4$ segundos, $p<0,02$, respectivamente). Conclusão: Hipertensos apresentam piores níveis de aptidão física comparados aos normotensos, e a prática de atividade física durante o período de seis meses não foi suficiente para diminuir a diferença entre esses grupos.

Descritores: hipertensão; aptidão física; exercício.

\section{ABSTRACT}

Introduction: Hypertension is a major risk factor for morbidity and mortality caused by cardiovascular diseases. Studies indicate an inverse relationship between mortality rate and physical fitness level. Objective: To determine if there are differences in physical fitness between hypertensive and normotensive individuals and whether there is an effect of regular physical activity on physical fitness in hypertensive similar to that found in normotensive individuals. Methods: The study included 214 women ( $\geq 40$ years), who were part of a physical activity program for 6 months. All subjects were submitted to an anthropometric and physical fitness assessment (AAHPERD). Results: Hypertensive individuals have a worse initial condition in relation to agility ( $G H$ initial mean $=24.2 \pm 0.4 \mathrm{sec}$. and $G N=20.9 \pm 0.8 \mathrm{sec}$., $p<0.01$ ), coordination (GH initial mean $=17.5 \pm 0.7 \mathrm{sec}$. and GN initial mean $=11.5 \pm 1.2 \mathrm{sec} ., p<0.01$ ) and aerobic endurance (GH initial mean $=568.5 \pm 12.2 \mathrm{sec}$. and GN initial mean $=506.8 \pm 21.7 \mathrm{sec} ., p<0.02)$. After 6 months of regular physical practice, hypertensive individuals continued to present worse results regarding agility, coordination and aerobic endurance (mean GH6 months $=22.9 \pm 0.4 \mathrm{sec}$. and mean GN 6 months $=19.97 \pm 0.7$ seconds; $p<0.01 ;$ GH 6 months $=16.1 \pm 0.7 \mathrm{sec}$ and GN 6 months $=10.6 \pm 1.3$ seconds, $p<0.01 ; G H 6$ months $=498.9 \pm 20.2 \mathrm{sec}$, and GN 6 months $=555.7 \pm 11.4$ seconds $p<0.02$, respectively). Conclusion: Hypertensive individuals have worse levels of physical fitness compared to normotensive and the practice of physical activity during the six-month period was not enough to decrease the difference between groups.

Keywords: hypertension; physical fitness; exercise.

\section{RESUMEN}

Introducción: La hipertensión es un factor de riesgo de gran importancia para la morbilidady la mortalidad causadas por enfermedades cardiovasculares. Los estudios muestran una relación inversa entre la tasa de mortalidad y el nivel de aptitud física. Objetivo: Determinar si existen diferencias en la aptitud física entre hipertensos y normotensos y se hay un efecto de la actividad física regular en la aptitud física en pacientes hipertensos similar al que se encuentran en sujetos normotensos. Métodos: Participaron del estudio 214 mujeres ( $\geq 40$ años), que participaron durante seis meses de un programa de actividad física. Todas fueron sometidas a evaluación antropométrica y evaluación de la aptitud física (AAHPERD). Resultados: Los sujetos hipertensos tienen peor estado inicial, con respecto a la velocidad (GH promedio inicial $=24,2 \pm 0,4$ seg. y $G N=20,9 \pm 0,8$ seg., $p<0,01$ ), la coordinación (GH promedio inicial $=17,5 \pm 0,7$ seg.y GN promedio inicial $=11,5 \pm 1,2$ seg., $p<0,01$ ) y la resistencia aeróbica (GH promedio inicial $=568,5 \pm 12,2 \mathrm{seg}$. y GN promedio inicial $=506,8 \pm 21,7 \mathrm{seg} ., p<0,02$ ). Después de seis meses de actividad física regular, los individuos hipertensos siguen teniendo peores resultados con respecto a la agilidad, coordinación y resistencia aeróbica (GH 6 meses promedio $=22,9 \pm 0,4$ seg. y GN 6 meses promedio $=19,97 \pm 0,7$ seg., $p<0,01$; GH6meses $=16,1 \pm 0,7$ seg.y GN6meses $=10,6 \pm 1,3$ seg., $p<0,01 ;$ GH6meses $=498,9 \pm 20,2$ seg.y GN 6 meses $=555,7 \pm 11,4$ seg. 
$p<0,02$, respectivamente). Conclusión: Los hipertensos tienen niveles de aptitud física peores en comparación con los normotensos y la práctica de la actividad física durante el perío do de seis meses no fue suficiente para disminuir la diferencia entre los grupos.

Descriptores: hipertensión; aptitud física; ejercicio.

\section{INTRODUÇÃO}

A hipertensão arterial tem sido indicada como o fator de risco conhecido de maior importância para a morbidade e mortalidade precoces causadas por doenças cardiovasculares.Considerada pela Organização Mundial de Saúde como o principal fator de morte no mundo ${ }^{1}$, a hipertensão também se configura como um problema de saúde pública no Brasil2. A prevalência da hipertensão arterial é de 20\% a 30\% na população brasileira, chegando a $50 \%$ nas idades mais avançadas².

É consenso, entre pesquisadores, que programas regulares de atividade física são atualmente reconhecidos como fator necessário na terapia de pacientes hipertensos. A recomendação em relação à atividade física é baseada em diversos estudos, os quais indicam que o exercício físico contribui para a diminuição da pressão arterial em repouso de indivíduos hipertensos ${ }^{3-5}$. Blair et al. ${ }^{6}$ verificaram uma redução em $64 \%$ na taxa de mortalidade em indivíduos que melhoraram seu nível de aptidão cardiorespiratória de baixa para a classificação moderada.

Sabendo que a falta de atividade física piora o nível de aptidão, e é fator de risco para hipertensão arterial ${ }^{7}$, torna-se necessário avaliar em quais aspectos relacionados à aptidão física o indivíduo hipertenso é diferente do normotenso.

A partir dessa realidade, o objetivo deste trabalho foi verificar se há diferença na aptidão física entre hipertensos e normotensos. Além disso, foi verificado se há efeito da prática regular de atividade física sobre a aptidão física em hipertensos similar àquelas encontradas em normotensos. Tais achados poderão indicar se existem e quais componentes de aptidão física estão limitados em decorrência da doença. Isso facilitará a adequação de um programa de atividade física visando à prevenção e melhora da condição de indivíduos.

\section{MATERIAL E MÉTODOS}

Participaram desse estudo 214 mulheres, com idades acima de 40 anos, que fizeram parte, por no mínimo seis meses, do Programa de Atividade Física para a Terceira Idade (PROFIT) e do Programa de Atividade Física para Diabéticos, Hipertensos e Obesos (oferecido gratuitamente pela parceria entre UNESP e a Fundação Municipal de Rio Claro-SP-Br).

Os indivíduos foram divididos em dois grupos: Hipertensos $(\mathrm{GH}$, $n=120)$ e Normotensos ( $G N, n=94)$. Essa classificação foi feita através do resultado da anamnese aplicada nos indivíduos previamente à prática de atividade física regular.

Este estudo foi submetido e aprovado ao Comitê de Ética em Pesquisa do Instituto de Biociências da Universidade Estadual Paulista, campus de Rio Claro, registrado em protocolo número 7823. Além disso, os procedimentos utilizados seguiram os padrões éticos da Declaração de Helsinki de 1975, revisada em 2008. Todas as informações sobre o estudo foram dadas aos sujeitos e um termo de consentimento livre e esclarecido, para participação no estudo, foi preenchido e assinado por estes. O estudo em questão respeita as normas internacionais de experimentação com humanos.

Os critérios de inclusão para participar do estudo foram: fazer parte do grupo de Atividade Física por pelo menos seis meses; ser mulher, ter recomendação médica para a prática de atividade física e ter idade igual ou acima de 40 anos.
As atividades foram realizadas duas ou três vezes por semana, de acordo com os princípios de periodização do treinamento físico. Cada sessão era composta por 60 minutos de atividades cardiorespiratórias (caminhadas e atividades lúdicas) e exercícios que trabalhavam os componentes força, agilidade, equilíbrio, flexibilidade e coordenação.

As atividades cardiorrespiratórias foram desenvolvidas mediante caminhadas curtas de intensidade moderada ou longas de intensidade leve, além de incluir jogos pré-desportivos e atividades lúdicas ou cooperativas, que envolviam deslocamentos com possíveis movimentações dos membros superiores.

As atividades foram orientadas com a finalidade de não superar a intensidade moderada. Os parâmetros utilizados para controle da intensidade formam o monitoramento da frequência cardíaca, até $70 \%$ da frequência cardíaca de reserva ${ }^{8}$, a escala de esforço subjetivo percebido de Borg (12 a 13 pontos na escala de 6 a 20) e o número de passos por sessão (2000 a 4000 no pedômetro). O emprego de um ou dois desses critérios de monitoramento, na maioria dos casos, é suficiente para o controle da intensidade desejada.

A sessões eram divididasem parte inicial, destinada à realização dos exercícios de alongamentos (5 a 10 min), a parte principal (40 a 45 min) à realização das atividades cardiorrespiratórias e neuromotoras e a parte final a atividades de volta à calma (5 a 10 min).

É importante ressaltar que rotineiramente foram realizadas aferições de pressão arterial no início das sessões. Caso o usuário se encontrasse com a pressão arterial (PA) igual ou superior a 160x90 mmHg o mesmo não participava da aula.

Todos os pacientes que se engajaram no programa foram submetidos a uma avaliação para obtenção de medidas antropométricas e funcionais, além de responderem a anamnese, aplicada previamente à primeira aula no programa.

A massa corporal foi verificada mediante a utilização de uma balança antropométrica com precisão de $100 \mathrm{~g}$. A estatura foi obtida por meio de um estadiômetro de madeira com escala de 0,1 cm.

Para avaliar o nível de aptidão física das participantes foi utilizado a bateria de teste da American Alliance for Health, Physical Education, Recreation and Dance (AAHPERD), formada por cinco testes (flexibilidade, coordenação, agilidade, força e resistência aeróbica), que foram desenvolvidos a fim de medir o nível de aptidão física de idosos.

Para verificar se existem alterações decorrentes da doença frente às capacidades física utilizou-se a comparação dos resultados obtidos em cada teste motor de indivíduos ativos hipertensos e normotensos.

\section{Análise estatística}

A comparação das médias entre o grupo de normotensos e hipertensos foi realizada utilizado-se o teste $t$ de Student para medidas independentes (STATISITICA 6.0).

Para comparar as avaliações iniciais e após seis meses de prática de atividade física, utilizamos a análise de variância (ANOVA) para medidas repetidas.

\section{RESULTADOS}

Como podemos verificar na Tabela 1, através do teste ANOVA, o IMC apresentou diferença significativa entre os dois grupos nas duas avaliações 
demonstrando que o IMC dos indivíduos hipertensos é maior e que a atividade física não diminuiu essa diferença mesmo após seis meses de prática.

Na Tabela 2 estão apresentados os resultados dos grupos de indivíduos hipertensos e indivíduos normotensos nos cinco testes que compõem a bateria da AAPHERD em duas situações (médias \pm desvios padrões): no início do programa e após seis meses de prática de atividade física.

Os resultados do teste flexibilidade $(\mathrm{cm})$ para os dois grupos e para os dois momentos indicam que para esse componente de aptidão não houve diferença entre os grupos em nenhuma das situações e que a prática de atividade física não alterou os valores dessa variável.

É possível perceber a diferença, estatisticamente significativa, em relação a agilidade entre o grupo de indivíduos hipertensos e normotensos para as duas situações de avaliação. Além de serem diferentes em ambas as situações, os dados acima demonstram que após seis meses de atividade física, ambos os grupos melhoram sua performance no teste de agilidade. Entretanto, mesmo havendo melhora, o grupo de indivíduos hipertensos não melhora a ponto de ter performances semelhantes com as do grupo de indivíduos normotensos.

Para o componente coordenação, como mostrado na Tabela 2, os dois grupos mostram-se diferentes tanto na avaliação inicial, quanto após seis meses de prática de atividade física. Ao comparar a avaliação inicial com a avaliação de seis meses, a ANOVA não apontou diferença significativa $(p=0,08)$.

Para os valores iniciais de força o grupo de indivíduos hipertensos não apresentou diferença estatisticamente significativa quando comparado com o grupo de indivíduos normotensos. Mesmo após seis meses de atividade física, não foram encontrados efeitos do treinamento para melhora da força em ambos os grupos.

Através da ANOVA encontramos diferença entre os grupos nas duas situações de avaliação, inicial e após seis meses de prática de atividade física. Ao comparar a avaliação inicial com a avaliação de seis meses depois, a ANOVA não apontou diferença significativa, demonstrando que mesmo após seis meses de atividade física não foram encontrados efeitos do treinamento para melhora da resistência aeróbia em ambos os grupos.

Em nenhum dos componentes de aptidão física a ANOVA apontou interação.

Tabela 1. Comparação de média e desvio padrão entre os participantes do grupo hipertenso e normotenso para as características antropométricas.

\begin{tabular}{c|c|c|c|c}
\hline & \multicolumn{2}{|c|}{ Inicial (Média + DP) } & \multicolumn{2}{c}{6 Meses (Média \pm DP) } \\
\cline { 2 - 5 } & Normotensos & Hipertensos & Normotensos & Hipertensos \\
\hline Peso $(\mathrm{Kg})$ & $66,7 \pm 2,2$ & $71,0 \pm 1,3$ & $66.7 \pm 2,1$ & $70,7 \pm 1,3$ \\
\hline Estatura $(\mathrm{m})$ & $1,55 \pm 3,7$ & $1,51 \pm 2,4^{*}$ & $1,55 \pm 3,8$ & $1,51 \pm 2,5^{*}$ \\
\hline IMC $\left(\mathrm{Kg} / \mathrm{m}^{2}\right)$ & $26,6 \pm 1,4$ & $29,5 \pm 1,7 *$ & $27,6 \pm 0,9$ & $30,4 \pm 0,5^{*}$ \\
\hline
\end{tabular}

Tabela 2. Comparação de Média + Desvio Padrão entre os grupos hipertensos e normotensos, para cada componente de aptidão física no início e após 6 meses de prática de atividade física.

\begin{tabular}{c|c|c|c|c}
\hline & \multicolumn{2}{|c|}{ Inicial (Média \pm DP) } & \multicolumn{2}{c}{6 Meses (Média \pm DP) } \\
\cline { 2 - 5 } & Normotensos & Hipertensos & Normotensos & Hipertensos \\
\hline Flexibilidade (cm) & $56,4 \pm 2,0$ & $56,9+1,1$ & $56,8 \pm 2,2$ & $58,5+1,2,9$ \\
\hline Agilidade (seg) & $20,9 \pm 0,8$ & $24,2 \pm 0,4^{*}$ & $19,97 \pm 0,7^{\dagger}$ & $22,9 \pm 0,4^{* \dagger}$ \\
\hline $\begin{array}{c}\text { Coordenação } \\
\text { (seg) }\end{array}$ & $11,5 \pm 1,2$ & $17,5 \pm 0,7^{*}$ & $10,6 \pm 1,3$ & $16,1 \pm 0,7^{*}$ \\
\hline Força (rep) & $26,3 \pm 1$ & $24,1 \pm 0,5$ & $25,7 \pm 0,9$ & $25,27 \pm 0,5$ \\
\hline Res. aeróbica (seg) & $506,8 \pm 21,7$ & $568,5 \pm 12,2^{*}$ & $498,9 \pm 20,2$ & $555,7 \pm 11,4^{*}$ \\
\hline${ }^{*}$ Diferença estatisticamente significativa entre grupos $p<0,05 ;{ }^{*}$ Diferença estatisticamente significativa comparando \\
resultado inicial e após 6 meses de atividade fisica (p<0,05).
\end{tabular}

\section{DISCUSSÃO}

Em relação a avaliação antropométrica, percebemos que indivíduos hipertensos avaliados possuem IMC superiores aos dos indivíduos normotensos. Isso ocorre tanto na avaliação inicial, quanto após seis meses de prática de atividade física. Segundo a VI Diretrizes Brasileiras de Hipertensão Arterial $^{2}$ a obesidade é fator de risco para a doença. A Organização Mundial de Saúde também afirma que o excesso de massa corporal é um fator para a predisposição da hipertensão, podendo ser responsável por 20\% a 30\% dos casos de hipertensão arterial ${ }^{9}$.

Para os resultados da avaliação da aptidão física, a resistência aeróbica, agilidade e coordenação apresentaram valores piores para mulheres hipertensas quando comparados com normotensas e essa diferença se manteve mesmo após seis meses de prática de atividade física, ou seja, indivíduos hipertensos continuam apresentando resultados piores.

Na literatura, o componente mais avaliado entre grupos de indivíduos normotensos e hipertensos é a resistência aeróbica. Segundo Carnethon et al. ${ }^{10}$ possuir baixo nível de aptidão cardiorrespiratória está associado com um risco entre 1.5 a 2.2 maior de desenvolver hipertensão quando comparados com indivíduos com aptidão em níveis ideais. Rankinen et al. ${ }^{11}$ encontraram uma forte associação entre hipertensão arterial e baixos níveis de aptidão cardiorrespiratória. Segundo seus resultados, altos níveis de aptidão física, avaliados somente pelo componente cardiorrespiratório (resistência aeróbica), foram associados a um baixo risco de desenvolver hipertensão arterial e que para cada 1\% na melhora da aptidão cardiorrespiratórias ocorre uma redução aproximada de 19\%no risco de surgimento da doença. O autor afirma que a aptidão cardiorrespiratória é um significante preditor do risco de desenvolvimento da hipertensão.

Níveis de aptidão cardiorrespiratória baixos podem interferir diretamente nas atividades de vida diária desses pacientes e como verificado por Dayi et al. ${ }^{12}$, durante as atividades diárias a fadiga é comum em pacientes com hipertensão e que isso ocorre mesmo quando baixos níveis de exercícios são exigidos. Lim et al. ${ }^{13}$ afirmam que a capacidade de exercício é 30\% menor em pacientes hipertensos se comparados com normotensos. Outro estudo realizado por Amery et al. ${ }^{14}$, reportou uma redução do consumo máximo de oxigênio de 18\% atribuído à hipertensão arterial.

Em nosso estudo, para os componentes força e flexibilidade, não foram encontrados diferença entre os grupos de mulheres hipertensas e normotensas. Já no quesito força muscular Artero et al. ${ }^{15}$ avaliaram 1.506 homens hipertensos com idades acima de 40 anos nos componentes força muscular e aptidão cardiorrespiratória. Os resultados demonstraram que homens hipertensos com níveis de forca muscular mais elevada apresentavam de 36\%-41\% menos risco de morte por todas as causas quando comparados com o grupo com menores níveis de força muscular. $\mathrm{O}$ autor conclui que altos níveis de força muscular estão associados a menores riscos de morte por todas as causas em homens hipertensos e que bons níveis de força muscular apresentam efeito protetivo contra o risco de morte em indivíduos hipertensos e recomenda que homens hipertensos se engajem em exercícios físicos com o objetivo de melhorar a força muscular. Outro importante estudo realizado por Yoon ${ }^{16}$, objetivou avaliar a associação entre hipertensão arterial e componentes da aptidão física em 9.216 mulheres Coreanas. Entre diversas variáveis avaliadas, estavam a aptidão cardiorrespiratória e a aptidão motora coordenativa que foram mensuradas através de testes de resistência muscular, flexibilidade, potência, agilidade, equilíbrio e força muscular. Os resultados encontrados demonstraram que as mulheres com pressão arterial normal apresentaram melhores resultados para massa corporal, níveis de aptidão cardiorrespiratória, aptidão motora coordenativa quando comparadas com mulheres pré-hipertensas ou hipertensas. A partir dos achados, o autor concluiu que níveis baixos de aptidão nos componentes agilidade, equilíbrio e 
aptidão cardiorrespiratória estão significativamente relacionados com o surgimento da hipertensão arterial e recomenda que para prevenir o surgimento da doença torna-se necessário a prática de exercício físico regular não somente para melhora da aptidão cardiorrespiratória, mas também a aptidão motora coordenativa.

No presente trabalho, outro objetivo importante foi verificar se existiu efeito após seis meses de prática de atividade física e se as diferenças encontradas entre os grupos, após esse tempo, eram atenuadas. Os componentes: coordenação, resistência aeróbica, força e flexibilidade, não apresentaram melhora nem piora, mas sim manutenção, dos valores da avaliação inicial para a avaliação após seis meses. Já o componente agilidade apresentou uma melhora, ou seja, uma redução no tempo de execução da tarefa, demonstrando que a prática de atividade física por seis meses produz melhoras na agilidade de mulheres com idades acima de 40 anos.

A literatura reporta declínios em todos os componentes da aptidão física. Por exemplo, entre os 25 e 65 anos de idade há diminuição de massa magra de 10 a 16\% por causa das perdas na massa óssea, no músculo esquelético e na água corporal total que acontecem com o envelhecimento ${ }^{17}$. Em relação à coordenação, a partir dos 40 anos parece ocorrer uma redução na qualidade coordenativa. Os movimentos coordenados na idade avançada apresentam uma velocidade diminuída, dificuldades de combinação de movimentos, diminuição da qualidade de execução ${ }^{18}$. Já a flexibilidade, com a idade adulta, perde significativa quantidade. Matsudo et al. ${ }^{17}$ em um estudo que objetivava avaliar as alterações no perfil neuromotor e capacidade funcional de mulheres ativas no período de um ano, verificou-se que, mesmo não havendo diferenças significativas nos valores de aptidão física, a prática regular de atividade física provoca manutenção de um nível de aptidão física que permite boa qualidade de vida durante processo de envelhecimento.

Através de nossos achados, podemos perceber que quatro dos cinco componentes de aptidão física tiveram seus níveis mantidos, e um deles, no caso a agilidade, apresentou melhora em decorrência da prática de seis meses de atividade física. Caminhando contra o processo de envelhecimento e dos efeitos da doença hipertensão arterial.

\section{CONCLUSÃO}

A partir dos dados analisados nesse trabalho, Indivíduos hipertensos apresentam piores níveis de aptidão física quando comparados com indivíduos normotensos. Não houve evolução da força, coordenação, resistência aeróbica e flexibilidade, sendo o componente agilidade o único a apresentar melhora através do treinamento. Entretanto, somente o fato de existir manutenção desses resultados, visto que os indivíduos estão em processo de envelhecimento, já é um grande benefício.

A prática de atividade física durante o período de seis meses não foi suficiente para diminuir a diferença entre os grupos de indivíduos normotensos e hipertensos. Esse diferença se mantém ao longo do tempo, mesmo praticando atividade física.

Por fim, não é possível estabelecer nexo causal entre componentes de aptidão física e o surgimento de hipertensão arterial. Não é possível estabelecer se os baixos valores de aptidão física, particularmente dos componentes aeróbico, coordenação e agilidade, são causadores da hipertensão ou se são consequência da doença.

Todos os autores declararam não haver qualquer potencial conflito de interesses referente a este artigo.

CONTRIBUIÇÕES DE AUTORES: Cada autor contribuiu individual e significantemente para o desenvolvimento deste artigo. LBB (0000-0002-5504-4102)* foi responsável pela redação do artigo, revisão, coleta dos dados e análise dos dados. EK (0000-0002-9404-3444)* realizou a análise estatística, contribuiu com o conceito intelectual do artigo e confecção de todo o projeto de pesquisa. ${ }^{*} \mathrm{ORCID}$ (Open Researcher and Contributor ID).

\section{REFERÊNCIAS}

1. World Health Organization. World health statistics 2012. Geneva: World Health Organization; 2012

2. Sociedade Brasileira de Cardiologia, Sociedade Brasileira de Hipertensão, Sociedade Brasileira de Nefrologia. VI Brazilian Guidelines on Hypertension. Arq Bras Cardiol. 2010;95(Suppl1):1-51.

3. Halbert JA, Silagy CA, Finucane P, Withers RT, Hamdorf PA, Andrews GR. Theeffectiveness of exercise training in lowering blood pressure: a meta-analysis ofrandomised controlled trials of 4 weeks or longer. J Hum Hypertens. 1997;11(10):641-9.

4. Whelton SP, Chin A, Xin X, He J. Effect of aerobic exercise on blood pressure:a meta-analysis of randomized, controlled trials. Ann Intern Med. 2002;136(7):493-503.

5. Fagard RH. Exercise characteristics and the blood pressure response to dynamicphysical training. Med Sci Sports Exerc. 2001;33(Suppl6):S484-92.

6. Blair SN, Kohl HW 3rd, Barlow CE, Paffenbarger RS Jr, Gibbons LW, Macera CA. Changes in physical fitness and all-cause mortality. A prospective study ofhealthy and unhealthy men. JAMA. 1995;273(14):1093-8.

7. Thompson PD, Buchner D, Pina IL, Balady GJ, Williams MA, Marcus BH, et al. Exercise and physical activity in the prevention andtreatment of atherosclerotic cardiovascular disease: a statement from the Councilon Clinical Cardiology (Subcommittee on Exercise, Rehabilitation, and Prevention)and the Council on Nutrition, Physical Activity, and Metabolism (Subcommittee on Physical Activity). Circulation. 2003;107(24):3109-16.

8. Wilmore JH, Costill D. Fisiologia do esporte e do exercício. São Paulo: Manole; 2013.

9. World Health Organization. Obesity. Preventing and managing the global epidemic. Genebra: World Health Organization; 2000.
10. Carnethon MR, Evans NS, Church TS, Lewis CE, Schreiner PJ, Jacobs DR Jr, et al. Joint associations of physical activity and aerobicfitness on the development of incident hypertension: coronary artery riskdevelopment in young adults. Hypertension. 2010;56(1):49-55.

11. Rankinen T, Church TS, Rice T, Bouchard C, Blair SN. Cardiorespiratoryfitness, BMI, and risk of hypertension: the HYPGENE study. Med Sci Sports Exerc. 2007;39(10):1687-92.

12. Dayi SU, Terzi S, AkbulutT, Akgöz H, Tartan Z, Gürkan U, et al. Effect of acute blood pressure reduction on oxygen uptake kineticsat the onset of exercise in hypertensive patients. Jpn Heart J. 2004;45(5):799-805.

13. Lim PO, MacFadyen RJ, Clarkson PB, MacDonald TM. Impaired exercise tolerance in hypertensive patients. Ann Intern Med. 1996;124(1 Pt 1):41-55.

14. Amery A, Julius S, Whitlock LS, Conway J. Influence of hypertension on thehemodynamic response to exercise. Circulation. 1967;36(2):231-7.

15. Artero EG, Lee DC, Ruiz JR, Sui X, Ortega FB, Church TS, et al. A prospective study of muscular strength and all-cause mortality inmen with hypertension. J Am Coll Cardiol. 2011;57(18):1831-7.

16. Yoon JH, So WY. Associations of hypertension status with physical fitness variables in korean women. Iran J Public Health. 2013;42(7):673-80.

17. Matsudo SM, Matsudo VKR, Neto TLBD, AraujoTL.Evolução do perfilneuromotor e capacidade funcional de mulheres fisicamente ativas de acordocom a idade cronológica. Rev Bras Med Esporte. 2003;9 (6):365-76.

18. Gobbi S, Villar R, Zago AS. Bases teórico-práticas do condicionamento físico. Rio de Janeiro: Guanabara Koogan;2005. 